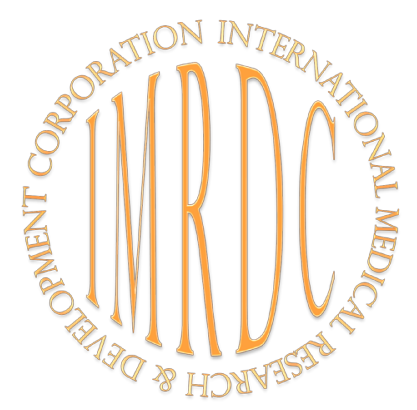

\title{
Art Therapy for Treating Anxiety in Adolescents with Psychosomatic Diseases
}

\author{
Zhanna Prokhorova, PhD*; Vladimir Polyakov, ScD; \\ Lyubov Rychkova, Corresponding Member of the RAS \\ Scientific Center for Family Health and Human Reproduction Problems \\ Irkutsk, the Russian Federation
}

\begin{abstract}
The purpose of our study was to develop and test a program for treating anxiety and hostility in adolescents with psychosomatic diseases (PSDs) by means of art therapy.

Methods and Results: This study included 20 patients with PSDs aged between 15 years and 17 years. The patients' examination included clinical data analysis, using standard records, and testing, using a questionnaire to reveal the severity of psychopathological symptoms (SCL-90-R, adapted by Tarabrina et al. 1997) and the Bekhterev Institute Personality Questionnaire (BIPQ, 1980). Art therapy sessions were conducted from 11:00 a.m. till 1:00 p.m., which coincides with the transition in the daily rhythm of autonomic nervous system activity and is more suitable for the reserve capabilities of the body. The course consisted of 10 daily 1.5 -hour procedures. The controlled study duration was $10 \pm 2$ days. The examination showed that adolescents with PSDs had an above-normal "Anxiety" (ANX) indicator, according to the SCL-90-R method, and a high score for the "Anxious" type (AT) of attitude toward the disease, according to the Bekhterev Institute Personality Questionnaire (BIPQ).

Conclusion: Art therapy for treatment of adolescents with PSD contributed to a normalization of their emotional state, helped them gain new experience in solving problems, and improved the quality of life. (International Journal of Biomedicine. 2020;10(4):453-456.)
\end{abstract}

Key Words: psychosomatic diseases $\bullet$ anxiety $\bullet$ adolescents $\bullet$ art therapy

\section{Introduction}

Currently, psychosomatic diseases (PSDs) are quite common among adolescents. Treatment of these diseases is not an independent medical discipline; it is an approach that takes into account a variety of causes that have led to the disease. ${ }^{(1,2)}$ According to the World Health Organization (WHO), 38\% to $42 \%$ of people in this age group apply to general clinics because of this disorder. A number of authors point to the role of increased anxiety in the development of PSD. ${ }^{(3,4)}$

In the scientific literature, anxiety is defined in different ways. According to one approach, anxiety is an emotional state. According to another one, it is a personality trait characterized by stability and a relative invariability throughout a person's

*Corresponding author: Zhanna Prokhorova, PhD, Senior Research Officer; Laboratory of Neuropsychosomatic Children's Pathology, Scientific Centre for Family Health and Human Reproduction Problems.Irkutsk, Russia.E-mail.proxorowa.janna2011@yandex.ru life. But at the heart of both, according to Spielberger, Khanin, and Kislovskaya, there is the expectation of a threat to a person's own personality. This can lead to constant stress, depletion of bodily resources, and hostility toward others, ${ }^{(5-8)}$ which hinders effective treatment.

It should be noted that according to current literature, one's self-concept is formed during the teen years. ${ }^{(9,10)}$ This means that in these years, knowledge about ourselves, other people and the world around us as a whole (cognitive component) is formed, and on this basis, a certain attitude and behavior toward the above is established (affective and behavioral components). Therefore, it is important to correct anxiety in adolescents with PSD to prevent an unfavorable emotional state from turning into a stable personality trait.

Such diseases require more than pharmaceutical treatment, because medication is not enough to cope with the disease completely; relapses will follow; therefore, the treatment should be accompanied by professional psychological work on the problem. 
Many authors consider that this is possible using art therapy, which allows, in a short period of time, adolescents with PSD to improve their psycho-emotional state, to show and realize their emotions, to get a socially acceptable experience of their expression and control, etc. ${ }^{(11-13)}$

The purpose of our study was to develop and test a program for treating anxiety and hostility in adolescents with PSD by means of art therapy.

\section{Materials and Methods}

This study included 20 patients aged between 15 years and 17 years: 5 of them suffered from vegetative-vascular dystonia and arterial hypertension, 5 had bronchial asthma, 5 - arthritis, and 5 - neurodermatitis. The diagnoses were verified on the basis of findings obtained as a result of clinical, laboratory and functional studies, as well as a psychological examination.

The patients' examination included clinical data analysis, using standard records, and testing, using a questionnaire to reveal the severity of psychopathological symptoms (SCL90-R, adapted by Tarabrina et al. 1997) and the Bekhterev Institute Personality Questionnaire (BIPQ, 1980). Art therapy sessions were conducted from 11:00 a.m. till 1:00 p.m., which coincides with the transition in the daily rhythm of autonomic nervous system activity and is more suitable for the reserve capabilities of the body. The course consisted of 10 daily 1.5hour procedures. Controlled study duration was $10 \pm 2$ days.

The study was approved by the Ethics Committee of the Scientific Center for Family Health and Human Reproduction Problems. Written informed consent was obtained from each research participant (or the participant's parent/guardian).

Statistical analysis was performed using the statistical software package SPSS version 21.0 (IBM Corp., Armonk, N.Y., USA). Continuous variables were presented as mean \pm standard deviation (SD). The Wilcoxon criterion was used to compare the differences between the paired samples. Pearson's correlation coefficient (r) was used to determine the strength of the relationship between the two continuous variables. A value of $P<0.05$ was considered significant.

\section{Results and Discussion}

In the first stage of the study, we diagnosed anxiety in adolescents with PSD and attempted to find its origin. It was discovered that they had an above-normal "Anxiety" (ANX) indicator, according to the SCL-90-R method, being equal to 1.08 and the "Anxious" type of attitude to the disease, according to BIPQ, being equal to 5 points (Fig.1).

Our participants were probably anxious and suspicious about their disease and possible complications. However, this is connected not with real complaints, such as high blood pressure, weakness, headaches, heart pains, etc., but with their focus on interpersonal relationships. This is confirmed by the strong negative correlation between ANX and Somatization (SOM) scales $(\mathrm{r}=-1, P<0.01)$, as well as the strong positive correlation between ANX and Interpersonal Sensitivity (INT) ( $\mathrm{r}=1, P<0.01$ ), according to the SCL-90-R method, AT, according to the BIPQ, and INT, according to the SCL-90-R method ( $\mathrm{r}=1, P<0.01$ ) (Fig.2). Based on the characteristics of adolescence described by V. Mukhina, ${ }^{(12)}$ and the presence of a disease that imposes certain restrictions on young people, we believe that their worries may be associated with the desire to be like their peers, who, for example, can walk around in their free time and eat fast food, while the subjects have to stay in bed and keep to a special diet, take medications, and undergo medical procedures. Their desire to be independent and separated is accompanied by knowing that they do not have their own means to purchase medicines, sometimes lack the strength and ability to take care of themselves on their own, and have to follow the doctors' prescriptions.

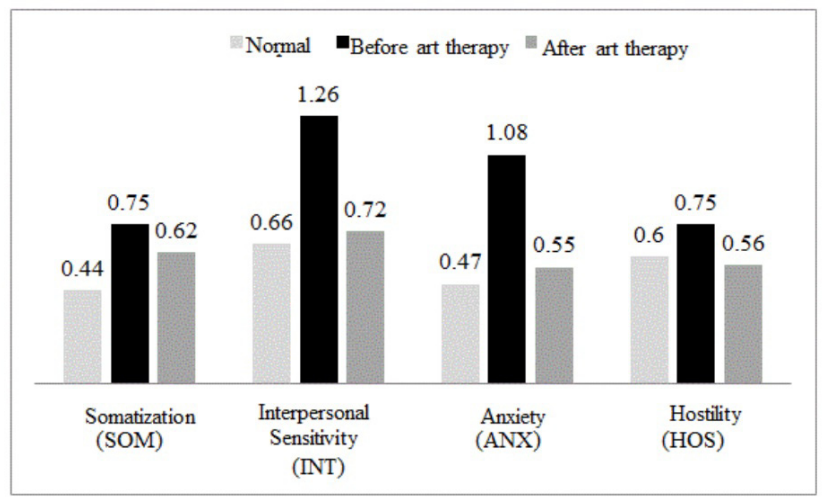

Fig. 1. Histogram of the severity of individual psychopathological symptoms revealed using the SCL90-R method before and after art therapy (the results are presented as mean score).

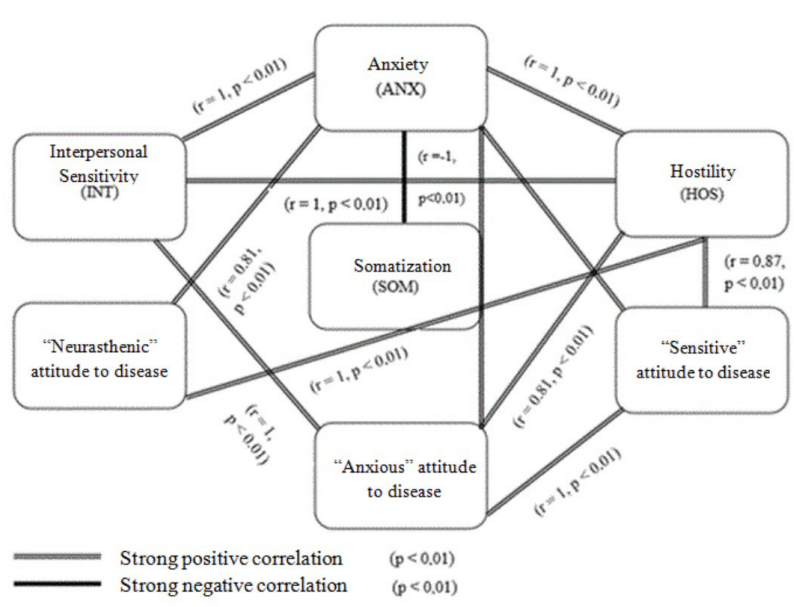

Fig. 2. Correlation pleiade

Besides, in our opinion, the need for hospital treatment and the inability to imitate the lifestyle of their peers not only creates tension in the body due to illness, but also anxiety about their own inferiority and the unwillingness of other people to communicate with them. This process is evidenced by the strong correlation between ANX, according to the SCL90-R method, and the "Sensitive" type (ST) of attitude to the disease, according to the BIPQ ( $\mathrm{r}=1, P<0.01)$ (Fig. 2). It is 
most likely due to the importance of intimate and personal communication with other adolescents and the mental polarity of those surrounding our subjects, who can be either kind or very cruel. This means that they can criticize them, or spread rumors about the disease, while subjects of this age, according to V. Mukhina, ${ }^{(12)}$ are very insecure.

Apart from interacting with peers, adolescents with PSD may experience anxiety about their own "inferiority" in relations with their parents. This is evidenced by the second diagnosed type of attitude toward the disease, according to the BIPQ, ST - 4 points (Fig.3). Perhaps the subjects believe that they will bring difficulties to their people; for example, the parents will have to spend time on accompanying them to procedures, and money on medicines for them.

Based on the above, the subjects may show irritability and anger toward others. In our opinion, such a reaction most likely occurs due to weakness, severe physical discomfort, treatment failures and unfavorable examination data, pain and resentment for being sick while others are healthy, fear of becoming dependent on others and not returning to the lifestyle and plans existing before the onset of the disease. This opinion is confirmed by the last type of attitude toward the disease, according to the BIPQ, revealed in the subjects"Neurasthenic" - 4.5 points - and the strong correlation between the scale "Hostility" (HOS) and INT, according to the SCL-90-R method ( $\mathrm{r}=1, P<0.01$; Fig. 2), as well as HOS, according to the SCL-90-R method, and ST according to the BIPQ (r=0.87, $P<0.01)$ (Fig.3).

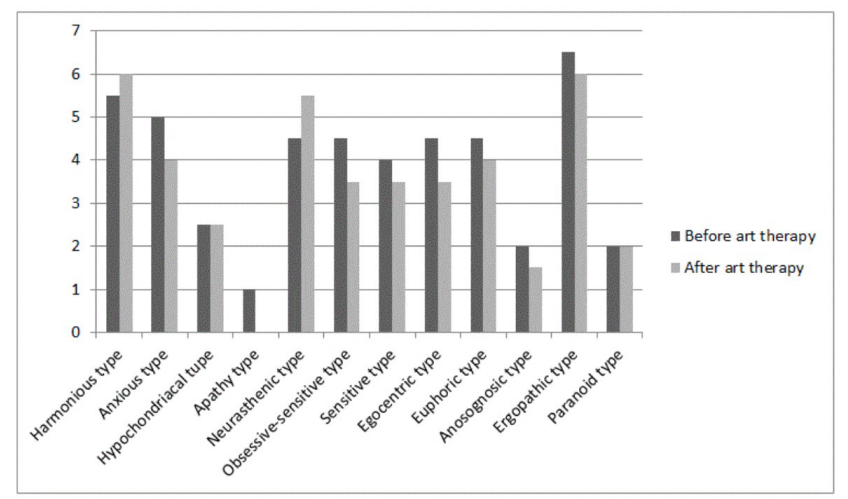

Fig. 3. Histogram of average values of attitude towards the disease according to BIPQ before and after art therapy

In the second stage, we conducted 10 group art therapy sessions for 1.5 hours each. The number of meetings and duration of sessions was chosen while taking into account inpatient treatment conditions of the subjects.

In the beginning, the participants introduced themselves and said a few words about themselves. Then the collaboration rules were introduced, and they did an exercise where everyone had the opportunity to individually communicate with all the other members of the group in turn, in order to show what could and couldn't be done when interacting with them. Thus, we tried to bring the subjects closer together and create a trusting contact between them.
Art therapy itself began with working on one's own name (the "Name" technique), since everyone had one, and this created a certain support for the subjects. In addition, it allowed them to discover many personal resources depending on different forms of their names.

Then we worked on the most difficult feelings for each participant, and all participants shared with the group their thoughts, conclusions and emotions arising from such feelings. This made it possible to re-experience and better understand them, gain experience of socially acceptable expression and relieve psycho-emotional stress.

In this connection, at the third stage we analyzed the presence of changes in the results obtained using the Wilcoxon rank sum test after art therapy and found no statistically significant differences $(\mathrm{z}=0.014, P<0.05)$. We can assume that the psychological factors in the development of PSD in the subjects were acquired a long time ago, have a more complex mechanism, and require more prolonged therapy.

However, quantitative and qualitative analysis shows that the ANX indicator, according to the SCL-90-R method (1.08 before and 0.55 after art therapy), and the score for AT, according to the BIPQ ( 5 before and 4 after art therapy), decreased and was next to normal. In our opinion, these results are due to the fact that the therapeutic work of the subjects in a group of peers with the same disease helped them share their experiences and learn that others were experiencing similar difficulties, which contributed to an understanding that their difficulties were normal, and enabled them to talk about their ways of coping with anxiety, i.e. relieve emotional stress and gain new experience in solving problems.

Thus, quantitative and qualitative analysis show changes in ANX, according to the SCL-90-R method, and the number of points for AT, in accordance with the BIPQ, after art therapy; however, these findings were not confirmed using the mathematical statistical methods $(\mathrm{z}=0.014, P<0.05)$. Due to the short duration of the art therapy course (10-12 sessions), the obtained results are probably short-term; therefore, in the future we plan to increase the duration of art therapy and check its effect on anxiety in adolescents with PSD, which will improve their quality of life and adaptive capabilities, and increase their level of social maturity, all of which may reduce the risk of their socio-psychological maladjustment in the future. The study results can be applied in the work of practical psychologists and in psychological counseling practice.

\section{Competing Interests} interests.

\section{References}

1. Kolesnikova LI, Dolgikh VV, Dzyatkovskaya EN, Polyakov VM. [Features of psychosomatic status in preschool and school-age children]. Bulletin of the East Siberian Scientific Center SBRAMS;2003;23(2):17-23. [Article in Russian].

2. Kolesnikova L, Dzyatkovskaya E, Rychkova L, Polyakov V. New approaches to identifying children of psychosomatic disorders risk group. Procedia - Social and Behavioral 
Sciences;2015:882-889.

3. Alekseeva EM, Ammosova AM. [Psychological rehabilitation of children and adolescents with increased anxiety against the background of psychosomatic diseases]. Medical rehabilitation in pediatric practice: achievements, conferences with international participation, 01.07.2013, Yakutsk, M. K. Ammosov North-Eastern Federal University [Electronic medium]. Edited by Prof. NV Savvina. Kirov: International Center for Research Projects; 2013;2(13):26-30. [Article in Russian].

4. Korabelnikova EA. [Anxiety disorders in adolescents]. Medical Council; 2018;18:34-42. [Article in Russian].

5. Kolesnikova LI, Dzyatkovskaya EN, Dolgikh VV, Polyakov VM, Rychkova LV. Adaptive development strategy for preserving the health of schoolchildren. M; 2015:176. [In Russian].

6. Mendelevich VD, Solovyova LM. Neurosology and psychosomatic medicine. M.: MEDpress-inform; 2002. [In Russian].

7. Roshchenko TA, Radionov GA. [Features of emotional disorders in adolescents with various psychosomatic diseases]. Problems of Theory and Practice of Modern Psychology [Electronic resource]: Materials of the XVIII annual All-Russian conference with international participation. 2019:323-325. Available at https:// iddin 1.ru/content/photo/novaapapka/psihologi/dara19/ problemyteoriiipraktikisovremennojpsihologii2019 compressed.pdf [Article inj Russian].

8. Schachter S, Singer J. Cognitive, social and psychological determinants of emotional state. In: Parrot WG, editor. Emotions in social psychology. Essential readings. Philadelphia: Psychology Press; 2001:76-93.

9. Thoits PA. Emotion norms, emotion work, and social order. Feelings and emotions: The Amsterdam symposium. N.Y.: Cambridge Univ. Press; 2004:359-379. Thoits PA. Emotion Norms, Emotion Work, and Social Order. In Manstead ASR, Frijda N, Fischer A, editors. Studies in emotion and social interaction. Feelings and emotions: The Amsterdam symposium. Cambridge University Press. 2004:359-378.

10. Leyens JP, Paladino PM, Rodriguez-Torres R, Vaes J. The Emotional Side of Prejudice: The Attribution of Secondary Emotion to Ingroups and Outgroups. Personality and Social Psychology Review. 2000;4:186-197.

11. Artyukhina NV. Psychological support of personality development by means of art therapy. Textbook. Odessa; 2015:138. [In Russian].

12. Mukhina VS. Age-related psychology. Phenomenology of development: Textbook. 10th Edition, Revised and Enlarged. M.: “Academy"; 2006:410-475. [In Russian].

13. Lebedeva LD. Art therapy practice: approaches, diagnostics, training system. SPb.: Rech; 2003:256. [In Russian]. 